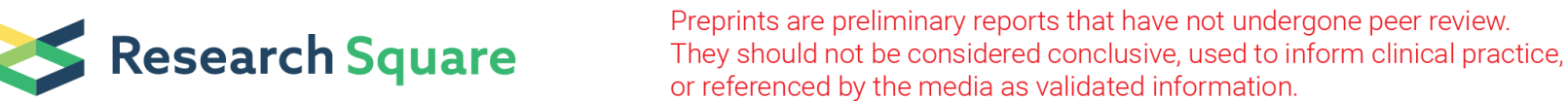

\section{The Effects of Mechanical Insufflation-Exsufflation on Lung Function and Complications in Cardiac Surgery Patients: A Pilot Study}

Meng-Fang Wu

Chang Gung Memorial Hospital

Tsai-Yu Wang ( $\nabla$ wang5531@gmail.com)

Chang Gung Memorial Hospital, Chang Gung University, School of Medicine https://orcid.org/00000001-8213-8393

\section{Da-Shen Chen}

Chang Gung Memorial Hospital

\section{Hsiu-Fong Hsiao}

Chang Gung Memorial Hospital

\section{Han-Chuang Hu}

Chang Gung Memorial Hospital

\section{Fu-Tsai Chung}

Chang Gung Memorial Hospital

\section{Ting-Yu Lin}

Chang Gung Memorial Hospital

Shu-Min Lin

Chang Gung Memorial Hospital

\section{Research article}

Keywords: Physiotherapy, Cardiac Surgery, Complications, Lung Functions, Mechanical InsufflationExsufflation, Atelectasis

Posted Date: July 30th, 2020

DOl: https://doi.org/10.21203/rs.3.rs-21076/v2

License: (c) (1) This work is licensed under a Creative Commons Attribution 4.0 International License. Read Full License

Version of Record: A version of this preprint was published at Journal of Cardiothoracic Surgery on December 1st, 2021. See the published version at https://doi.org/10.1186/s13019-021-01738-x. 


\section{Abstract}

Background: Postoperative positive pressure lung expansion is associated with decreased pulmonary complications and improved clinical outcomes. The aim of the present study was to compare the differences in post-operative pulmonary complications and clinical outcomes between two groups of study subjects who underwent cardiac surgery; one included subjects who received mechanical insufflation-exsufflation (MI-E) and the other included subjects who received intermittent positive pressure breathing (IPPB) therapy.

Methods: This retrospective study included 51 subjects, who underwent cardiac surgery in an intensive care unit of a tertiary hospital during the time period from June 2017 to February 2018. After liberation from mechanical ventilation, the subjects received lung expansion therapy by means of two types of positive pressure devices, MI-E $(n=21)$ or IPPB $(n=30)$. The pulmonary complications, lung function, and clinical outcomes were compared between the two groups.

Results: Subjects in both groups displayed similar baseline characteristics and underwent similar types of surgical procedures. Compared to subjects who received non-oscillatory therapy, those who received MI-E therapy had higher post-operative force vital capacity $(58.4 \pm 4.74 \%$ vs. $46.0 \pm 3.70 \%, p=0.042)$, forced expiratory volume in one second $(62.4 \pm 5.23 \%$ vs. $46.8 \pm 3.83 \%, p=0.017)$, and peak flow rate $(67.1 \pm 5.53 \mathrm{~L}$ vs. $55.7 \pm 4.44 \mathrm{~L} p=0.111)$. However, the incidence of chest pain was higher in the MI-E group $(n=13,61.9 \%)$ than in the IPPB group $(n=4,16.7 \%$; odds ratio, $0.123,95 \%$ confidence interval, $0.03-0.45$; $\mathrm{p}=0.002$ ). The length of hospital and ICU stay, development of atelectasis, pneumonia, and pleural effusion were similar in both the groups.

Conclusion : Both IPPB and MI-E therapies have similar effects on preventing post-operative complications in cardiac surgery patients. However, compared to IPPB therapy, MI-E therapy was associated with better-preserved pulmonary function and higher incidence of chest pain.

\section{Background}

Literature reveals that the incidence of postoperative pulmonary complications (PPCs), subsequent to in cardiac surgery, ranges from $1.9 \%$ to $7.9 \%[1,2]$, which include prolonged hospital stay or postoperative morbidity and mortality.[3] Recently, the European joint taskforce guidelines for perioperative clinical outcome (EPCO) considered respiratory infection, respiratory failure, pleural effusion, atelectasis, pneumothorax, bronchospasm and aspiration pneumonitis as the composite measures of PPCs.[4] Changes to the respiratory system occur immediately on induction of general anesthesia: respiratory drive and muscle function are altered, lung volume reduced, and atelectasis developed in majority of patients receiving a neuromuscular blocking agent. Post-operative sputum retention is a common phenomenon. General anesthesia, especially with a tracheal tube, causes impairment of the mucociliary transport in the airways and this effect may persist during the postoperative period. This combination of 
reduced FRC, residual atelectasis, ineffective cough and abnormal respiratory control generates an ideal situation for the development of PPCs [5].

Although evidence that supports the use of respiratory physiotherapy in hospitalized patients is controversial [6], this type of intervention is widely used in clinical practice [7]. A wide range of techniques of respiratory physiotherapy are available, in order to assist lung expansion and facilitate the clearance of pulmonary secretions. A variety of therapeutic interventions can be performed using the physical abilities of the physiotherapist and/or the patient, such as deep breathing exercises, manual pulmonary clearance techniques and forced expiration techniques [8]. Other types of therapeutic interventions, such as breathing exercises with positive pressure, require specific devices. Several treatment modalities, such as prophylactic continuous positive airway pressure ventilation (CPAP) have been shown to reduce PPCs.[9] A randomized controlled trial reported that supplementary therapy using bilevel positive airway pressure (BiPAP), in addition to the protocols, significantly reduced the incidence of atelectasis.[10] Moreover, intermittent positive pressure breathing (IPPB) is also a popular method for the prevention of atelectasis after cardiac surgery. The primary purpose of using positive pressure devices for breathing exercises is to facilitate lung expansion and thus prevent the development of atelectasis and to improve the clearance of secretions.

Cough plays a critical role in airway clearance. Cough augmentation techniques, including lung volume recruitment or mechanically assisted cough, are used to prevent respiratory complications associated with muscle weakness [11]. Mechanical insufflation-exsufflation (MI-E) consists of lung insufflation by means of positive pressure, followed by an active negative-pressure exsufflation, which simulates a cough and moves the pulmonary secretions towards the mouth [11]. MI-E is generally used in patients with neuromuscular diseases or muscle weakness, caused by injuries to the central nervous system [12]. Moreover, MI-E has also been used to facilitate airway mucus clearance in mechanically ventilated ICU patients [13]. Considering the theoretical benefits of providing positive pressure for lung expansion and facilitation of sputum clearance through cough augmentation, it can be safely suggested that post-operative use of MI-E in cardiac surgery patients can aid in the prevention of PPCs. Therefore, the aim of the present study was to compare the effects of MI-E with that of IPPB, pertaining to lung expansion and subsequent prevention of lung atelectasis, after cardiac surgery.

\section{Methods}

\section{Study population}

This retrospective study included 51 subjects, who underwent cardiac surgery in Chang Gung Memorial Hospital, a tertiary hospital in Taiwan, during the time period from June 2017 to February 2018. Subjects who had a history of previous thoracic surgery, thoracic abnormalities and subjects younger than 18 years of age were excluded from the study. The Chang Gung Medical Foundation Institutional Review Board approved the study and waived the requirement for informed consent, owing to the retrospective nature of this study. 


\section{Study design}

The medical records of all the subjects included in the current study were reviewed, in order to extract data regarding the clinical characteristics and laboratory results. Additionally, information regarding the baseline characteristics, comorbidities, pulmonary function tests, the type of cardiac surgery performed, the type of IPPB used (IPPB or oscillatory IPPB); the duration of ventilator use; the duration of ICU stay and hospital stay and the type of PPCs encountered was collected.

One day prior to the surgery, all subjects were given instructions regarding breathing techniques such as deep diaphragmatic ventilation and effective coughing, by two respiratory therapists. Postoperatively, all the subjects received mechanical ventilation (pressure-controlled, peak airway pressure of $25 \mathrm{~cm} \mathrm{H}_{2} \mathrm{O}$, targeting a tidal volume of $8 \mathrm{~mL} / \mathrm{kg}$, an initial positive end-expiratory pressure (PEEP) of $10 \mathrm{~cm} \mathrm{H}_{2} \mathrm{O}$, with a fraction of inspired oxygen $\left(\mathrm{FiO}_{2}\right)$ level designed to keep arterial oxygen saturation at $\left.>95 \%\right)$. Weaning was initiated if the subjects were normothermic and hemodynamically stable, and operative revision was not required. Weaning was facilitated by reducing the respiratory rate and changing to pressure support ventilation if spontaneous breathing had resumed. Subjects were extubated if they were fully conscious, responsive to commands, had sufficient protective airway reflexes and met the following criteria: partial pressure of oxygen $>70 \mathrm{~mm} \mathrm{Hg}$ at an $\mathrm{FiO}_{2}$ of 0.35 and a PEEP of $7 \mathrm{~cm} \mathrm{H}_{2} \mathrm{O}$; and partial pressure of carbon dioxide $<46 \mathrm{~mm} \mathrm{Hg}$ at a pressure support of $<8 \mathrm{~cm} \mathrm{H}_{2} \mathrm{O}$. On extubation, two respiratory therapists guided the implementation of either IPPB (ResMed's Astral 150) or MI-E (Philips E70® Cough Assistor) therapy. The lung expansion therapies were performed 3 times a day and started within 8 hours after extubation. In all the subjects, the peak airway pressure, ranging from 10 to $25 \mathrm{~cm} \mathrm{H}_{2} \mathrm{O}$, was adjusted during each session, resulting in widening of the thoracic cage. The simultaneous attainment of deep inspiration at the peak pressure confirmed the satisfactory effect of respiratory therapy. The target of respiratory therapy was to attain more than ten satisfactory effects during each session.

All the subjects underwent pulmonary function tests (PFT) prior to the surgery. After performing either IPPB or MI-E therapy for five days, all the patients received a follow-up pulmonary function test measurement. Two measurements of PFT were taken and the better result was recorded. Two independent pulmonologists, blinded to the group allocation in the study, analyzed postero-anterior (PA) chest X-ray films of subjects, which were taken before surgery and thereafter daily up to the fifth postoperative day. Atelectasis, lobar collapse, pulmonary infiltrations, and pleural effusions were recorded.

\section{Definitions}

The definitions of PPCs, used in the current study, were in accordance with the European perioperative clinical outcome (EPCO).[5] In brief, the definition of atelectasis was lung opacification with mediastinal shift, hilum or hemidiaphragm shift towards the affected area, with compensatory hyperinflation in the adjacent non-atelectatic lung. Pleural effusion was defined as CXR with the radiographic feature of blunting of the costophrenic angle, loss of sharp silhouette of the ipsilateral 
hemidiaphragm in upright position, displacement of adjacent anatomical structures, or (in supine position) hazy opacity in one hemithorax with preserved vascular shadows. The diagnostic criteria for pneumonia was CXR with at least one of the following radiographic features: infiltrate, consolidation, cavitation; plus at least one of the following clinical features: fever $>38^{\circ} \mathrm{C}$ with no other evident cause, white cell count $<4$ or $>12^{\star} 10^{9} \mathrm{~L}^{-1}$, subjects of age greater than 70 years with altered mental status with no other evident cause; plus at least two of the following features: new purulent/ changed sputum, increased secretions/suctioning, new/worse cough/dyspnea/tachypnea, rales/bronchial breath sounds, worsening gas exchange.

\section{Statistical analysis}

Data were expressed as mean \pm SEM (standard error of the mean). Two-tailed Student's $t$ test was used to compare the continuous variables in the two groups, while Mann-Whitney test was used for the nonnormal distributions. Categorical variables were compared by means of the $\mathbf{x}^{2}$ or Fisher's exact tests. $\mathbf{P}$ value of less than 0.05 was considered to be statistically significant. Analysis was carried out using SPSS (version 13.0; SPSS; Chicago, IL) statistical software.

\section{Results}

\section{Demographics and clinical characteristics of subjects}

The current study involved a total of 48 subjects, who underwent cardiac surgery and received either IPPB or MI-E therapy in our hospital, during the time period from June 2017 to February 2018. 21 (41.2\%) of the 51 subjects received oscillatory IPPB (MI-E therapy). The baseline demographics and clinical characteristics of the study subjects are listed in Table 1. The study subjects were divided into two groups based on the type of respiratory therapy received: the MI-E group and the IPPB group. The mean age of the subjects in the MI-E and IPPB groups were 63.8 years and 62.5 years, respectively. The proportion of male subjects in the MI-E and IPPB groups were $66.7 \%$ and $56.7 \%$, respectively. Other characteristics including BMI, habit of smoking, type of surgery, maximal inspiratory pressure, duration of ventilator use and the duration of ICU stay were similar between the two groups.

\section{Comparison of post-operative changes in lung function between the two groups}

The lung function changes in both groups are shown in Table 2. The forced expiratory volume in one second $\left(\mathrm{FEV}_{1}\right)$ and forced vital capacity $(\mathrm{FVC})$ were observed to decrease after surgery, in both groups. The ratio of $\mathrm{FEV}_{1} / \mathrm{FVC}$ was within the normal range; indicating a restrictive pulmonary defect after surgery. The percentage of predictive value of post-operative FVC $(58.4 \pm 4.74$ vs. $46.0 \pm 3.70 \%, p=0.042)$, and $\mathrm{FEV}_{1}(62.4 \pm 5.23$ vs. $46.8 \pm 3.83 \%, \mathrm{p}=0.017)$ were significantly inferior in the IPPB group, compared to the MI-E group. In addition, the reductions of FVC, $\mathrm{FEV}_{1}$, and PEF from preoperative to postoperative were significantly smaller in the MI-E group, compared to the IPPB group (Figure 1D). Therefore, pulmonary 
functions were better preserved in the subjects who received post-operative MI-E therapy, compared to those who received IPPB therapy.

\section{Complications in the two groups}

The incidence of post-operative complications including pneumonia, atelectasis and pleural effusion were observed to be similar in both the groups (Table 3). However, the incidence of chest pain was significantly higher in the MI-E group, compared to the IPPB group (MI-E vs. IPPB group, $61.9 \%$ vs. $16.7 \%$; OR, $0.123 ; 95 \%$ C.I., $0.03-0.45 ; p=0.002$ ). After five days of positive pressure therapy, the percentage of patients who showed improvement of atelectasis was similar in both the groups (MI-E vs. IPPB group, $61.9 \%$ vs. $50.0 \%$; OR, $0.62 ; 95 \%$ C.I., 0.20-1.91; $p=0.40$ ) (Table 4). Although the percentage of improvement of atelectasis in the MI-E group was higher, compared to the IPPB group, in subjects with segmental or lobar atelectasis, the difference was not statistically significant.

\section{Discussion}

The present study demonstrated that cardiac surgery patients who received MI-E therapy had better lung function compared to those who received IPPB therapy. The incidence of post-operative pulmonary complications including pneumonia, atelectasis, and pleural effusion, were similar in both the groups. However, the number of subjects who experienced chest pain was higher in the MI-E group, compared to the IPPB group.

Compatible with previous reports [14], the current study observed significant decline in the values of effort-dependent lung function tests, such as FVC, $\mathrm{FEV}_{1}$, and peak expiratory flow rate, after surgery in both groups. The study subjects developed a proportional decrease in lung volume without change in the $\mathrm{FEV}_{1} / \mathrm{FVC}$ ratio; indicating a restrictive pulmonary defect after surgery. The normal activity of most of the respiratory muscle groups is impaired after a major surgery, including the airway muscles, abdominal muscles, and diaphragm [14]. Factors contributing to this dysfunction include anesthetic agents and neuromuscular blocking agents, drugs used for post-operative analgesia, pain, disturbed sleep patterns and the inflammatory response to surgery. In addition to the simple muscle weakness, the etiology of decreased lung function may involve poor sputum clearance, poor co-ordination between muscle groups and failure of the normal physiological reflexes, which control the effort-dependent lung function. Theoretically, MI-E therapy may simulate coughing and facilitate better sputum clearance in patients. In the present study, the percentage of improvement of segmental and lobar atelectasis in the MI-E group was observed to be higher, compared to the IPPB group. However, the difference was not statistically significant; probably due to the inadequate sample size. Therefore, further research is mandatory to elucidate the effects of MI-E therapy on the improvement of atelectasis and lung function.

Several studies have demonstrated that pulmonary complications are more common than cardiac complications, in patients undergoing cardiac surgery $[2,5]$. In patients who undergo cardiac surgery, the diminution in lung volume provokes atelectasis. Discoid atelectasis is apparently unavoidable, in the 
immediate postoperative phase. Pleural fluid has been shown to predispose to atelectasis [15]. The high occurrence of pleural effusions and discoid atelectasis leads to their coincidental appearance. In the present study, development of postoperative complications were observed to be similar between the two study groups; indicating that both types of respiratory therapy devices, which were used in the study, are equally effective in preventing post-operative complications. Several devices have been used to reduce post-operative pulmonary complications. IPPB was first introduced in the 1940s. [7] It became particular popular in 1980s and 1990s, before other devices such as CPAP, BiPAP and high-flow nasal cannula therapy became available in clinical practice. However, the role of IPPB is still controversial.[6, 16-20] Literature reports that the incidence of atelectasis in patients who received post-operative IPPB therapy ranges from $40 \%$ to $55 \%[17,20]$. The present study showed that $50 \%$ of subjects in the MI-E group and $42.9 \%$ of subjects in the IPPB group developed atelectasis, which was concurrent with the previous studies. The incidence of chest pain was significantly higher in the MI-E group, compared to the IPPB group. Due to similar peak airway pressure target and tidal volume setting in both the groups, a probable explanation for the increased incidence of chest pain may be the active negative-pressure exsufflation in MI-E. The MI-E therapy design may simulate a cough or even induce cough and move the secretions towards the mouth. Although the pain could be controlled by remedial medical treatment, caregivers should pay more attention to pain control in cardiac surgery patients, who receive post-operative MI-E therapy.

As is the case with any mechanical positive-pressure device, potential complications of in-exsufflation include abdominal distention, aggravation of gastroesophageal reflux, hemoptysis, chest and abdominal discomfort, acute cardiovascular effects, and pneumothorax. However, rarely have these been reported in literature [11]. Physiological effects on the cardiovascular system were studied during the early phases of development of in-exsufflation. The effects on cardiovascular system include an increase in the peripheral venous pressure (about one third more than during normal coughing), and slight increase in blood pressure [21]. Pulse can increase or decrease with in-exsufflation. Severe bradyarrhythmia has been reported in patients with high spinal cord injury and premature ventricular contractions have been reported in an adolescent with Duchenne muscular dystrophy [21, 22]. Prudent measures to avoid the complications of in-exsufflation include short rest breaks between applications of in-exsufflation; avoid hyperventilation and administration of in-exsufflation before meals or feedings, vigorous medical treatment of gastroesophageal reflux, and adequate treatment of any airway inflammation.

In spite of better improvement of postoperative pulmonary function in MI-E group, there was no clinical benefit noted for both therapies in terms of length of ICU stay and postoperative pulmonary complications. Therefore, the improvement of pulmonary function did not translate into a change in clinical outcomes. The similar clinical outcomes in both groups may be attributable to the frequent and careful attention to respiratory care that they received. All patients were coached to site at the edge of the bed and take deep breaths with every treatment. Early, supervised, frequent mobilization with coach to take deep breath with both devices resulted in similar incidences of postoperative complications and length of ICU stay in both groups. 
A major limitation of the present study is its retrospective nature, which may have led to bias in the selection of study subjects. Additionally, the sample size of the study is small, and therefore the results should be interpreted with caution. The results of this pilot study may raise the attention that using distinct devices for lung expansion therapy in post-operative patients may have different outcomes in lung function and complications. Therefore, a prospective study with a larger sample size is warranted to further confirm the clinical outcomes in patients receiving IPPB or MI-E therapy. Emerging evidence suggests that MI-E therapy is useful in a selected group of patients, such as those with muscle weakness and without airway instability [11]. Nevertheless, before its routine use in clinical practice, more studies are needed to investigate the safety and utility of MI-E therapy in the clearance of secretions from central and peripheral airways, as well as the impact on clinically relevant outcomes, including the incidence of ventilator-associated pneumonia, improvement of atelectasis, length of ICU/ hospital stay, and mortality.

\section{Conclusion}

Both IPPB and MI-E therapies have similar effects on the prevention of post-operative complications in cardiac surgery patients and both are reasonable choices for post-operative physiotherapy. MI-E therapy showed better improvement in pulmonary functions, but a higher incidence of chest pain, compared to IPPB therapy. However, further studies are required to assess the precise impact of MI-E therapy on postoperative complications.

\section{Abbreviations}

postoperative pulmonary complications (PPCs)

European perioperative clinical outcome (EPCO)

functional residual capacity (FRC)

continuous positive airway pressure ventilation (CPAP)

bilevel positive airway pressure (BiPAP)

intermittent positive pressure breathing (IPPB)

Mechanical insufflation-exsufflation (MI-E)

intensive care unit (ICU)

positive end-expiratory pressure (PEEP)

fraction of inspired oxygen $\left(\mathrm{FiO}_{2}\right)$

positive end-expiratory pressure (PEEP) 
pulmonary function tests (PFT)

cardiovascular surgeon (CVS)

postero-anterior (PA)

body mass index (BMI)

forced expiratory volume in one second $\left(\mathrm{FEV}_{1}\right)$

forced vital capacity (FVC)

peak expiratory flow (PEF)

\section{Declarations}

\section{Ethics approval and consent to participate}

The Chang Gung Medical Foundation Institutional Review Board : 201901803B0

Consent for publication

Not applicable

\section{Availability of data and materials}

The data that support the findings of this study are available from The Chang Gung Medical Foundation Institutional Review Board but restrictions apply to the availability of these data, which were used under license for the current study, and so are not publicly available. Data are however available from the authors upon reasonable request and with permission of The Chang Gung Medical Foundation Institutional Review Board.

\section{Competing Interests}

Conflict of interest statement: The authors declare that there is no conflict of interest.

\section{Funding}

This project was supported by the Minister of Science and Technology of Taiwan, R.O.C. (MOST 1082314-B-182A-130) and research grants from the Chang Gung Memorial Hospital, Taiwan (CIRPG3H0051). The funders had no role in the design of this study nor the collection, analysis, and interpretation of data or in the writing of this manuscript.

\section{Authors' contributions}


Literature search: TYW; HCH; FTC; TYL; SML; Data collection: MFW; DSC ; Study design: MFW; TYW; DSC; HFH; SML; Analysis of data: MFW; TYW; DSC; HFH; SML; Manuscript preparation: TYW; HCH; FTC; TYL; SML; Review of manuscript: TYW; SML (all authors have read and approved the manuscript)

\section{Acknowledgements}

This project was supported by the Minister of Science and Technology of Taiwan, R.O.C. (MOST 1082314-B-182A-130), research grants from the Chang Gung Memorial Hospital, Taiwan (CIRPG3H0051) and research grants from the Chang Gung Memorial Hospital, Taiwan (CMRPG3J0611)

\section{References}

1. Canet J, Gallart L, Gomar C, et al. Prediction of postoperative pulmonary complications in a population-based surgical cohort. Anesthesiology. 2010; 113(6):1338-50.

2. Mazo V, Sabate S, Canet J, et al. Prospective external validation of a predictive score for postoperative pulmonary complications. Anesthesiology. 2014; 121(2):219-31.

3. Guldner A, Kiss T, Serpa Neto A, et al. Intraoperative protective mechanical ventilation for prevention of postoperative pulmonary complications: a comprehensive review of the role of tidal volume, positive end-expiratory pressure, and lung recruitment maneuvers. Anesthesiology. 2015; 123(3):692713.

4. Jammer I, Wickboldt N, Sander M, et al. Standards for definitions and use of outcome measures for clinical effectiveness research in perioperative medicine: European Perioperative Clinical Outcome (EPCO) definitions: a statement from the ESA-ESICM joint taskforce on perioperative outcome measures. Eur J Anaesthesiol. 2015; 32(2):88-105.

5. Miskovic A, Lumb AB. Postoperative pulmonary complications. Br J Anaesth. 2017; 118(3):317-34.

6. Fiore JF, Chiavegato LD, Paisani DM, Colucci DB. Utilization of positive-pressure devices for breathing exercises in the hospital setting: a regional survey in Sao Paulo, Brazil. Respir Care. 2010; 55(6):719-24.

7. Denehy L, Berney S. The use of positive pressure devices by physiotherapists. Eur Respir J. 2001; 17(4):821-9.

8. Pasquina P, Tramer MR, Granier JM, Walder B. Respiratory physiotherapy to prevent pulmonary complications after abdominal surgery: a systematic review. Chest. 2006; 130(6):1887-99.

9. Zarbock A, Mueller E, Netzer S, Gabriel A, Feindt P, Kindgen-Milles D. Prophylactic nasal continuous positive airway pressure following cardiac surgery protects from postoperative pulmonary complications: a prospective, randomized, controlled trial in 500 patients. Chest. 2009; 135(5):12529.

10. Al Jaaly E, Fiorentino F, Reeves BC, et al. Effect of adding postoperative noninvasive ventilation to usual care to prevent pulmonary complications in patients undergoing coronary artery bypass grafting: a randomized controlled trial. J Thorac Cardiovasc Surg. 2013; 146(4):912-8. 
11. Homnick DN. Mechanical insufflation-exsufflation for airway mucus clearance. Respir Care. 2007; 52(10):1296-305; discussion 306-7.

12. Auger $C$, Hernando V, Galmiche H. Use of Mechanical Insufflation-Exsufflation Devices for Airway Clearance in Subjects With Neuromuscular Disease. Respir Care. 2017; 62(2):236-45.

13. Ferreira de Camillis ML, Savi A, Goulart Rosa R, et al. Effects of Mechanical Insufflation-Exsufflation on Airway Mucus Clearance Among Mechanically Ventilated ICU Subjects. Respir Care. 2018; 63(12):1471-7.

14. Braun SR, Birnbaum ML, Chopra PS. Pre- and postoperative pulmonary function abnormalities in coronary artery revascularization surgery. Chest. 1978; 73(3):316-20.

15. Gale GD, Sanders DE. The Bartlett-Edwards incentive spirometer: a preliminary assessment of its use in the prevention of atelectasis after cardio-pulmonary bypass. Can Anaesth Soc J. 1977; 24(3):40816.

16. Gale GD, Sanders DE. Incentive spirometry: its value after cardiac surgery. Can Anaesth Soc J. 1980; 27(5):475-80.

17. Iverson LI, Ecker RR, Fox HE, May IA. A comparative study of IPPB, the incentive spirometer, and blow bottles: the prevention of atelectasis following cardiac surgery. Ann Thorac Surg. 1978; 25(3):197200.

18. Pfenninger J, Roth F. Intermittent positive pressure breathing (IPPB) versus incentive spirometer (IS) therapy in the postoperative period. Intensive Care Med. 1977; 3(4):279-81.

19. Pasquina P, Tramer MR, Walder B. Prophylactic respiratory physiotherapy after cardiac surgery: systematic review. BMJ. 2003; 327(7428):1379.

20. Oikkonen M, Karjalainen K, Kahara V, Kuosa R, Schavikin L. Comparison of incentive spirometry and intermittent positive pressure breathing after coronary artery bypass graft. Chest. 1991; 99(1):60-5.

21. Bach JR. Update and perspective on noninvasive respiratory muscle aids. Part 2: The expiratory aids. Chest. 1994; 105(5):1538-44.

22. Miske LJ, Hickey EM, Kolb SM, Weiner DJ, Panitch HB. Use of the mechanical in-exsufflator in pediatric patients with neuromuscular disease and impaired cough. Chest. 2004; 125(4):1406-12.

\section{Tables}

Table 1: Baseline characteristics of the study subjects 


\begin{tabular}{|c|c|c|c|}
\hline ariables & Ml-E group ${ }^{*}, n=21$ & IPPB group ${ }^{\dagger}, n=30$ & p value \\
\hline je, years & $63.8 \pm 2.5$ & $62.5 \pm 2.3$ & .715 \\
\hline ale gender, $n(\%)$ & $14(66.7)$ & $17(56.7)$ & .472 \\
\hline $\mathrm{VI}, \mathrm{kg} / \mathrm{m}^{2}$ & $24.0 \pm 0.8$ & $24.7 \pm 0.7$ & .518 \\
\hline noker, n (\%) & $10(47.6)$ & $15(50)$ & .615 \\
\hline iabetes Mellitus, n (\%) & $10(47.6)$ & $11(38.1)$ & 917 \\
\hline ypertension, $\mathrm{n}(\%)$ & $10(47.6)$ & $17(56.7)$ & .524 \\
\hline Jronary arterial disease, n (\%) & $9(42.9)$ & $15(50.0)$ & .615 \\
\hline JPD, n (\%) & $1(4.8)$ & $1(3.3)$ & .796 \\
\hline sthma, n (\%) & $0(0)$ & $1(3.3)$ & 999 \\
\hline aronic renal disease, $\mathrm{n}(\%)$ & $9(42.9)$ & $12(40.0)$ & 838 \\
\hline pes of surgery & & & .933 \\
\hline horacic endovascular aortic repair & $3(14.3)$ & $3(10.0)$ & \\
\hline 'alve replacement surgery & $10(47.6)$ & $16(53.3)$ & \\
\hline oronary artery bypass grafting & $8(38.1)$ & $10(33.3)$ & \\
\hline eptal repair surgery & $0(0)$ & $1(3.3)$ & \\
\hline hite Blood Cell count & $9.7 \pm 1.2$ & $11.2 \pm 1.0$ & .333 \\
\hline eatinine & $1.4 \pm 0.3$ & $1.9 \pm 0.4$ & .407 \\
\hline jdium & $140.0 \pm 0.8$ & $139.3 \pm 0.8$ & .537 \\
\hline jtassium & $3.9 \pm 0.1$ & $4.0 \pm 0.1$ & .563 \\
\hline aximal inspiratory pressure, $\left(\mathrm{cm} \mathrm{H}_{2} \mathrm{O}\right)$ & $36.2 \pm 1.9$ & $37.4 \pm 1.7$ & .644 \\
\hline uration of ventilator use (days) & $2.1 \pm 0.4$ & $2.7 \pm 0.6$ & .453 \\
\hline ength of ICU§ stay (days) & $4.2 \pm 0.6$ & $4.8 \pm 0.8$ & .560 \\
\hline
\end{tabular}

MI-E: Mechanical insufflation-exsufflation

IPPB: Intermittent positive pressure breathing

BMl: Body mass index

ICU: Intensive care unit

COPD: Chronic obstructive pulmonary disease

Table 2: Comparison of post-operative changes in lung functions between the two groups 


\begin{tabular}{|l|c|c|c|c|}
\hline Variables & & MI-E* $^{*}$ group, $\mathrm{n}=21$ & $\mathrm{IPPB}^{\dagger}$ group, $\mathrm{n}=30$ & $\mathrm{p}$ value \\
\hline \multirow{3}{*}{$\mathrm{FVC}^{\ddagger}, \mathrm{L}$} & Preoperative & $2.19 \pm 0.16$ & $2.12 \pm 0.18$ & .806 \\
\cline { 2 - 5 } & Postoperative & $1.77 \pm 0.20$ & $1.39 \pm 0.11$ & .082 \\
\cline { 2 - 5 } & Difference & $0.41 \pm 0.14$ & $0.73 \pm 0.13$ & .108 \\
\hline \multirow{3}{*}{$\mathrm{FVC}^{\ddagger}, \%$} & Preoperative & $68.1 \pm 3.74$ & $67.4 \pm 4.85$ & .916 \\
\cline { 2 - 5 } & Postoperative & $58.4 \pm 4.74$ & $46.0 \pm 3.70$ & .042 \\
\cline { 2 - 5 } & Difference & $9.68 \pm 4.65$ & $21.4 \pm 3.61$ & .050 \\
\hline \multirow{2}{*}{$\mathrm{FEV}_{1} \S, \mathrm{L}$} & Preoperative & $1.78 \pm 0.13$ & $1.73 \pm 0.15$ & .804 \\
\cline { 2 - 5 } & Postoperative & $1.57 \pm 0.17$ & $1.12 \pm 0.08$ & .011 \\
\cline { 2 - 5 } & Difference & $0.21 \pm 0.12$ & $0.61 \pm 0.11$ & .017 \\
\hline \multirow{3}{*}{$\mathrm{FEV}_{1} \S, \%$} & Preoperative & $69.8 \pm 4.35$ & $69.4 \pm 4.96$ & .947 \\
\cline { 2 - 5 } & Postoperative & $62.4 \pm 5.23$ & $46.8 \pm 3.83$ & .017 \\
\cline { 2 - 5 } & Difference & $7.45 \pm 4.49$ & $22.6 \pm 3.65$ & .011 \\
\hline \multirow{2}{*}{$\mathrm{PEF}_{1} \S / \mathrm{FVC}^{\ddagger}, \mathrm{L}$} & Preoperative & $81.0 \pm 1.85$ & $85.6 \pm 1.95$ & .106 \\
\cline { 2 - 5 } & Postoperative & $90.3 \pm 4.24$ & $81.1 \pm 1.59$ & .025 \\
\cline { 2 - 5 } & Difference & $-9.32 \pm 3.61$ & $4.58 \pm 1.77$ & .001 \\
\cline { 2 - 5 } & Preoperative & $76.60 \pm 5.12$ & $82.9 \pm 5.75$ & .440 \\
\cline { 2 - 5 } & Postoperative & $67.1 \pm 5.53$ & $55.7 \pm 4.44$ & .111 \\
\cline { 2 - 5 } & Difference & $9.6 \pm 5.52$ & $27.2 \pm 4.32$ & .014 \\
\hline
\end{tabular}

MI-E*: Mechanical insufflation-exsufflation

$\mathrm{IPPB}^{\dagger}$ : intermittent positive pressure breathing

FVC: Forced vital capacity

$\mathrm{FEV}_{1} \S$ : Forced expiratory volume in one second

PEF"l: Peak expiratory flow

Table 3: Comparison of the incidence of post-operative complications between the two groups 


\begin{tabular}{|l|c|c|c|c|}
\hline Variables & Ml-E ${ }^{*}$ group, $n=21$ & IPPB $^{\dagger}$ group, $n=30$ & $\begin{array}{c}\text { OR } \\
\text { 95\% C.I. }\end{array}$ & P value \\
\hline Pneumonia & $1(4.8 \%)$ & $2(6.7 \%)$ & $\begin{array}{c}1.43 \\
(0.12-16.86)\end{array}$ & .777 \\
\hline Atelectasis & $13(61.9 \%)$ & $15(50.0 \%)$ & $\begin{array}{c}0.62 \\
(0.20-1.91)\end{array}$ & .402 \\
\hline -Segmental & $8(38.1 \%)$ & $13(43.3 \%)$ & $\begin{array}{c}1.24 \\
(0.39-3.88)\end{array}$ & .709 \\
\hline -Lobar & $5(23.8 \%)$ & $2(6.7 \%)$ & $\begin{array}{c}.229 \\
(0.04-1.32)\end{array}$ & .099 \\
\hline Pleural effusion & $8(38.1 \%)$ & $14(46.7 \%)$ & $\begin{array}{c}1.42 \\
(0.46-4.43)\end{array}$ & .544 \\
\hline Chest pain & $13(61.9 \%)$ & $4(16.7 \%)$ & $\begin{array}{c}0.123 \\
(0.03-0.45)\end{array}$ & .002 \\
\hline
\end{tabular}

MI-E*: Mechanical insufflation-exsufflation

$\mathrm{IPPB}^{\dagger}$ : Intermittent positive pressure breathing

OR $§$ : Odds ratio

C.I. ${ }^{\ddagger}$ : Confidence interval

Table 4: The percentage of improvement in atelectasis after respiratory therapy in post-cardiac surgery patients

\begin{tabular}{|l|c|c|c|c|}
\hline Percentage of improvement & MI-E ${ }^{*}$ group & $\mathrm{IPPB}^{\dagger}$ group & $\begin{array}{c}\mathrm{OR}^{\ddagger}, \\
95 \% \text { C.I. }\end{array}$ & P value \\
\hline Total atelectasis, N(\%) & $9 / 13(69.2)$ & $8 / 15(53.3)$ & $\begin{array}{c}1.97 \\
(0.42-9.32)\end{array}$ & .460 \\
\hline Segmental atelectasis, N(\%) & $8 / 8(100)$ & $8 / 13(61.5)$ & $\begin{array}{c}11.0 \\
(0.52-231)\end{array}$ & .111 \\
\hline Lobar atelectasis, N (\%) & $1 / 5(20.0)$ & $0(0)$ & $\begin{array}{c}1.67 \\
(0.05-58.3)\end{array}$ & .999 \\
\hline
\end{tabular}

MI-E*: Mechanical insufflation-exsufflation

$\mathrm{IPPB}^{\dagger}$ : intermittent positive pressure breathing 
$\mathrm{OR}^{\ddagger}$ : Odds ratio

C.I. §: Confidence interval

Figures

A

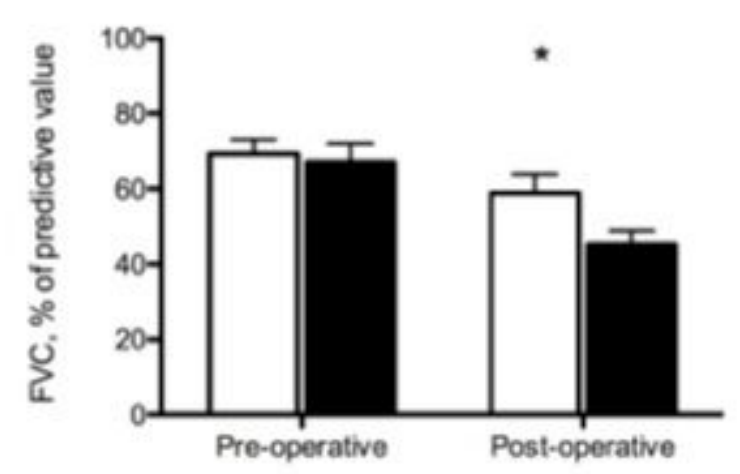

C

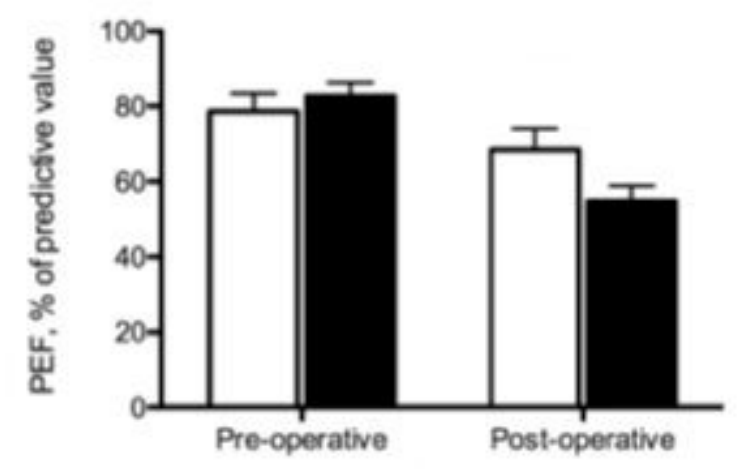

B

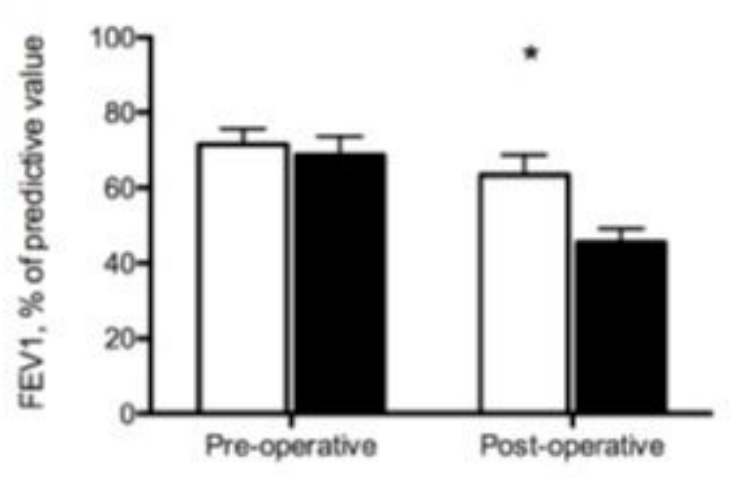

D

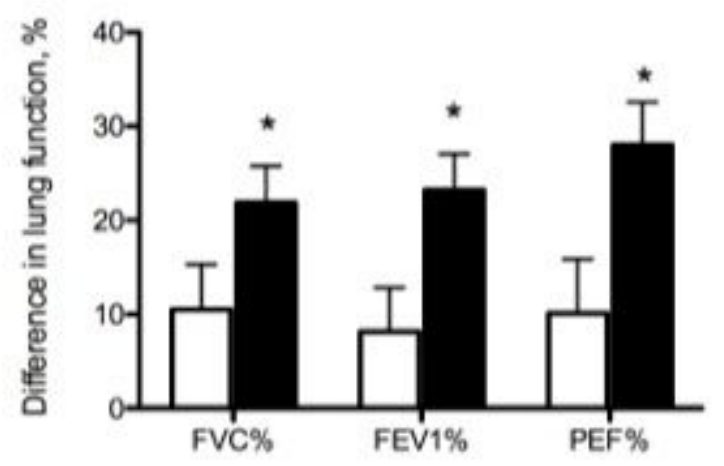

\section{Figure 1}

Comparison of lung functions between the subjects who received mechanical insufflation-exsufflation (MI-E) therapy and the subjects who received intermittent positive pressure breathing (IPPB) therapy (A) Forced vital capacity (FVC) of predictive value were significantly higher in subjects who received MI-E therapy (open bar), compared to the subjects who received IPPB therapy (black bar) (* indicates $p<0.05$ ); (B) Forced expiratory volume in one second (FEV1) of predictive value were significantly higher in subjects who received MI-E therapy (open bar), compared to the subjects who received IPPB therapy (black bar) (* indicates $\mathrm{p}<0.05$ ); (C) Peak expiratory flow rate (PEF) of predictive value were similar between both groups; (D) The difference between pre-operative and post-operative values of FVC, FEV1, 
PEF were significantly higher in subjects who received MI-E therapy (open bar), compared to the subjects who received IPPB therapy (black bar) (* indicates $\mathrm{p}<0.05$ ); (data expressed as mean \pm SEM) 